\title{
Improving EBSD precision by orientation refinement with full pattern matching.
}

\author{
Aimo Winkelmann ${ }^{1,2}$, B. Matat Jablon ${ }^{2}$, Vivian Tong ${ }^{3}$, Carol Trager Cowan ${ }^{2}$, Ken Mingard $^{3}$
}

1) Academic Centre for Materials and Nanotechnology, AGH University of Science and Technology, al. A. Mickiewicza 30, 30-059 Krakow, Poland

2) Department of Physics, SUPA, University of Strathclyde, Glasgow G4 ONG, United Kingdom

3) National Physical Laboratory, Teddington, Middlesex, TW11 OLW, United Kingdom

\begin{abstract}
We present a comparison of the precision of different approaches for orientation imaging using electron backscatter diffraction (EBSD) in the scanning electron microscope. We have used EBSD to image the internal structure of WC grains, which contain features due to dislocations and subgrains. We compare the conventional, Hough-transform based orientation results from the EBSD system software with results of a high-precision orientation refinement using simulated pattern matching at the full available detector resolution of $640 \times 480$ pixels. Electron channeling contrast imaging (ECCI) is used to verify the correspondence of qualitative $\mathrm{ECCl}$ features with the quantitative orientation data from pattern matching. For the investigated sample, this leads to an estimated pattern matching sensitivity of about $0.5 \mathrm{mrad}\left(0.03^{\circ}\right)$ and a spatial feature resolution of about $100 \mathrm{~nm}$. In order to investigate the alternative approach of post-processing noisy orientation data, we analyse the effects of two different types of orientation filters. Using reference features in the high-precision pattern matching results for comparison, we find that denoising of orientation data can reduce the spatial resolution, and can lead to the creation of orientation artefacts for crystallographic features near the spatial and orientational resolution limits of EBSD.
\end{abstract}

\section{Introduction}

Electron backscatter diffraction (EBSD) is a scanning electron microscope technique which can be used to map the microstructure of a material by identifying the phase and the orientation from electron backscatter diffraction patterns (EBSPs) formed at each point of the map [1]. EBSPs consist of multiple bands formed by Kikuchi diffraction of backscattered electrons from the different lattice planes within the interaction volume of the electrons. Conventionally, the diffraction bands are identified using a Hough transform which converts

This article has been accepted for publication and undergone full peer review but has not been through the copyediting, typesetting, pagination and proofreading process, which may lead to differences between this version and the Version of Record. Please cite this article as doi:

10.1111/jmi.12870.

This article is protected by copyright. All rights reserved. 
linear features into separated peaks in Hough space. Identifying and matching these peaks to the expected Kikuchi bands and their relative orientations enables the measurement of orientation and the discrimination between different crystalline phases. The angular resolution of this approach is limited by the required resolution in Hough space, however, with angular precisions typically stated to be of the order of $0.5^{\circ}$ for high-speed implementations [2]. For similar reasons, the discrimination between phases with closely related structures is limited by the resolution in Hough space. The angular and phase sensitivity both worsen if the EBSP size is reduced for high speed measurements by binning of pixels.

There has therefore been an increase in the development of other methods to improve on or complement the information available from EBSD measurements and to derive more information from them, as fast EBSD detectors increase the ease with which they can be captured. One approach is to post-process the orientation information delivered by the EBSD system, using for example Kuwahara filters [3] [4], bilateral filters [5], spline-based filters [6], variational methods from mathematical image analysis [7], or pattern averaging [8]. These filtering approaches can involve a loss of spatial information if operated on features with dimensions approaching the filter kernel size. Compared to the postprocessing of noisy orientation data, other approaches involve the direct comparison of the experimental EBSPs with theoretical patterns which can be simulated using different models for the underlying diffraction geometry and electron scattering effects. For example, discrimination of subtle phase differences such as polarity changes in non-centrosymmetric crystals has been shown to be possible by comparison with simulated patterns and by multivariate statistical analysis [9]. A dictionary approach to matching simulated patterns to acquired patterns has been reported to improve phase discrimination [10] and suggests a reduction in orientation noise [11]. Orientation refinement by full pattern matching (FPM) of simulated to measured EBSPs [12], [13] has been shown to improve angular resolution from the reduction in noise and improved identification of sub grains in quartzite, with an approximate improvement of precision by a factor of 3 . This method has been applied to silicon crystals in a separate study [14], where as a measure of angular resolution the $95^{\text {th }}$ percentile of the KAM angle distribution was obtained at $0.06^{\circ}$ [14]. An improvement in orientation precision when using a pattern matching approach for standard-resolution patterns has also been discussed with respect to the orientation data delivered by different EBSD systems [15]. Increased topographical and structural detail have been shown to be produced from the analysis of the EBSP background intensity and the channelling-in effects on this parameter [16].

For highest sensitivity to small relative lattice strains and rotations, cross-correlation techniques are used in high angular resolution EBSD (HR-EBSD) [17] [18] [19], which typically requires experimental Kikuchi patterns with resolutions in the megapixel range. In the scope of HR-EBSD, the application of simulated patterns has also been discussed as 
promising an absolute reference if several limiting factors could be accurately controlled [20].

This work presents further use of the refinement by pattern matching [12] to demonstrate improvement in angular precision by about an order of magnitude compared to orientation data delivered by the EBSD system software. In particular, the features revealed by this improved precision are shown to be dislocations by comparison with high resolution ECCI and it is shown that it is possible to quantify the misorientations around such features. The ECCl signal [21], [22], [23], [24], [25], [26], [27] is produced when a sample is placed so that a crystal plane or planes are at, or close to, the Bragg angle with respect to the incident electron beam. Any deviation in crystallographic orientation or in lattice constant due to local strain will produce a variation in contrast in the resultant ECCI micrograph. Extremely small changes in orientation and strain are detectable, revealing, for example, low angle tilt and rotation boundaries and atomic steps and extended defects such as dislocations. Techniques have also been developed to identify the dislocations revealed by ECCI [28], [29]. While ECCl can be used to image very small changes in misorientation, it does not provide quantitative information on the misorientations. High angular precision EBSD is therefore a very attractive technique to use together with $\mathbf{E C C l}$ in the study of extended defects [30] [31].

The increased orientation precision used here is obtained by post-processing of the same raw Kikuchi patterns as would be used in the conventional system analysis, thus involving no additional effort in data acquisition except for the storage of the Kikuchi patterns. As an application example, we studied the sub-structure and dislocations of selected grains in an as-sintered WC-Co hardmetal, combining qualitative $\mathrm{ECCl}$ and quantitative EBSD information. Hardmetals are widely used because of their high compressive strength and wear resistance as materials for tooling or machining [32]. Understanding their structure by high resolution methods in the SEM is of particular interest because of the difficulty of sample preparation of such high-density materials for the TEM. We compare the orientation precision of the full-pattern matching approach with approaches based on post-processing of orientation data by filtering techniques. We demonstrate that full-pattern orientation refinement reveals detailed fine structure which is drowned by orientation noise in the conventional analysis and which cannot be recovered by filtering operations. The resulting possible loss of spatial resolution is important to consider for applications which apply a filtering of noisy orientation data.

\section{Method}

\section{Material and EBSD acquisition}

The sample used in this work was a WC-11wt.\%Co coarse-grained hardmetal sample with a mean circle equivalent diameter grain size of $4.5 \mu \mathrm{m}$. A high-quality surface preparation 
was key to obtaining good ECCl prior to acquisition of EBSD maps; initial polishing used conventional metallographic processes, grinding with successively finer diamond grit and finishing with a 20 minute colloidal silica polish as the final stage. This was followed by Ar+ ion beam polishing in a Hitachi IM4000 Argon Ion Beam polishing system. Initial polishing was carried out at $6 \mathrm{kV}$ for 30 minutes to remove mechanical polishing damage, followed by lower beam energies of 3 and $1.5 \mathrm{kV}$. The sample was examined in a Zeiss Auriga60 scanning electron microscope.

The grains imaged in this study were chosen based on the proximity of the sample surface normal to the principle crystal directions [0001] and [11-20]. Suitably oriented grains were identified from a low magnification EBSD map of a large area. ECCI rotation series were acquired from each grain as described in reference [33]. We have checked that in the presented ECCI images, all dislocations are visible which have been found in the rotation series. The two grains were then mapped using EBSD, where an EBSP was acquired at each point on the map using an Oxford Instruments Nordlys F camera with Aztec software. Patterns were acquired with a $30 \mathrm{kV} 10 \mathrm{nA}$ electron beam at full camera resolution of $640 \times$ 480 pixels on a $37 \times 28 \mathrm{~mm}^{2}$ phosphor screen, using an exposure time of $60 \mathrm{~ms}$ without frame averaging. The working distance was $14.9 \mathrm{~mm}$ and the sample-phosphor distance was $20.1 \mathrm{~mm}$. The step size between patterns was $0.055 \mu \mathrm{m}$ for the [0001] grain and 0.050 $\mu \mathrm{m}$ for the [11-20] grain. An example EBSP is shown in Figure 1a. Patterns from the Co matrix were not considered in this study.

The initial orientations were determined from the saved patterns with two available Hough transform based approaches implemented in the Oxford Instruments Aztec software: firstly, using the conventional Hough Transform indexing (HT) with 8 detected bands, edge detection and a Hough resolution setting of 60 and, secondly, using the Oxford Instruments Refined Accuracy (RA) procedure with 12 detected bands and a Hough resolution of 90 . The RA procedure uses the HT solution as a seed, and iteratively refines this by fitting the simulated band edge shapes and positions to the experimental EBSP. RA accounts for the hyperbolic shape of the Kikuchi bands, whereas HT approximates them as straight lines [34]. RA includes an extra degree of freedom in detecting Kikuchi band positions, similar to the 3D Hough transform [35].

The FPM method was then applied to the saved patterns using starting orientations determined by the HT method. In order to study the influence of pattern resolution, fullpattern matching was also applied to the stored patterns after software pixel binning, which sums the pixel values in each bin to give $320 \times 240,160 \times 120$ and $80 \times 60$ pixel patterns. In this way, the electron dose per pattern is approximately maintained, while the solid angle per pixel is increased. 


\section{Pattern Matching}

The pattern matching approach we applied is described in detail in references [12] and [13]. In brief, each experimentally observed Kikuchi pattern is compared with simulated patterns which are reprojected according to the varying parameters of the crystal orientation and the fixed parameters of the projection geometry. For each measured EBSD map point, we use an optimization procedure to find the crystal orientation parameters which lead to the highest similarity between the simulated and the experimental pattern. The image similarity is measured by the normalized cross-correlation (NCC) coefficient [36]. The starting values of the optimization procedure are taken from the conventional analysis as provided by the manufacturer system software. In order to exclude systematic effects of these starting orientations, we applied random misorientations with a maximum misorientation angle of $1^{\circ}$ to the starting values. The optimization procedure finds the local maximum of the NCC reliably within approximately $2^{\circ}$ from the initial values. Convergence is checked by restarting the optimization from different initial values. The projection center (PC) as a function of map position was calibrated by optimizing both the orientation and the PC for patterns on a subgrid of $11 \times 11$ map points and then fitting the PC results to a linear transformation model as discussed in [14].

In the current investigation, we apply the pattern matching approach to patterns at full resolution of the EBSD hardware that was used, $640 \times 480$ pixels ( $300 k$ pixels), extending the previous studies with pattern resolutions of the order of $20 \mathrm{k}$ pixels (e.g. $160 \times 120$ ). In Figure 1, we show a comparison of (a) an experimental pattern from a WC grain with the corresponding (b) simulation determined by the FPM approach. In order to facilitate a reproducible pattern comparison, the patterns have been normalized to a mean of zero and unit standard deviation, including background removal as described in [37]. The NCC value of $r=0.69$ indicates a very good agreement between simulation and experiment. A major contribution to the remaining differences is excess-deficiency effects on the Kikuchi bands which are not considered in the present simulation model.

\section{Results}

We show results for measurements on two different WC grains, oriented with their surface normal approximately parallel to [0001] and [11-20], respectively.

\section{Reduction of EBSD Orientation Noise}

In Figure 2a, an initial image of the measured sample area containing the [0001] grain from the EBSD data is shown, which was calculated from the total intensity of the saved Kikuchi pattern [38]. This map mainly shows the topography of the sample. The orientation map in Figure $2 b$, showing the [0001] oriented grain at the centre, was produced using the standard Hough transform indexing method and is shown without post-processing except for removal of misindexed pixels within the binder phase region. As expected, with the displayed 
orientation based on an inverse pole figure colour scale which is not sensitive to small changes in orientation, the grains are uniform in appearance. The same sample region was also investigated by $\mathrm{ECCl}$, which is shown in Figure 2c. Features due to dislocations and subgrain boundaries can be clearly seen in the $\mathrm{ECCl}$ micrograph. For comparison, Figure $2 \mathrm{~d}$ shows the best-fit values of the NCC coefficient resulting from the refinement of the initial orientations by FPM. In this NCC map, we see contrast due to topography, contrast due to the subgrain boundary, and also some features which correlate with the dislocation structures seen in ECCl micrograph of Figure 2c. Obviously, the best-fit NCC values are measurably influenced by contributions other than changes in local crystal orientation, which can be used as a sensitive imaging signal as shown in Figure $2 \mathrm{~d}$. In the full pattern matching approach, we thus need to estimate the sensitivity of the optimization process to these orientation-independent contributions which are not specific to the local crystal orientation. For example, topographic effects, which influence the possible maximal NCC values, could also lead to slightly different optimized orientation values as a side effect.

The improvement of the orientation precision which we have obtained by the FPM approach is illustrated in Figure 3. We compare the precision of the orientations using various orientation data analysis and visualization approaches on the same set of saved Kikuchi patterns. As a well-known indicator of local orientation precision, we show the maps of the kernel average misorientation (KAM) angle of each map point to its nearest neighbours. We compare the conventional Hough-transform-based $(H T)$ analysis (a), the Refined Accuracy (RA) values (b), and the full-pattern matching (FPM) results for different levels of pattern binning, starting from $80 \times 60$ pixels (c), $160 \times 120$ (d), $320 \times 240$ (e), and the full detector resolution of $640 \times 480$ (f). Figure $3 \mathrm{~g}$ shows the corresponding KAM angle histograms for each of the maps. Comparison of the histograms illustrates that the map produced using the conventional fast HT analysis (Figure 3a) shows mainly noise, with a $95^{\text {th }}$ percentile at $\vartheta_{95}=0.28^{\circ}$. This value is improved significantly to $\vartheta_{95}=0.16^{\circ}$ using the additional RA analysis in the manufacturer software. The pattern matching results further improve the KAM angle distributions, with $\vartheta_{95}$ reduced to $0.13^{\circ}$ at $80 \times 60$, and to $0.05^{\circ}$ at $640 \times 480$. We note that the result at $2 \times 2$ binning for $320 \times 240$ pixels is $\vartheta_{95}=0.06^{\circ}$, which is already very close to the result for the full resolution. This is also visible in the KAM maps, which allow features due to subgrains and even dislocations to be clearly distinguished. In contrast, only a very rough delineation of the subgrain is seen in the HT and RA maps, which means that much of the orientation precision that is potentially available in a $640 \times 480$ pattern is restricted by the orientation noise of the data analysis approaches in the specific, speed-optimized, algorithms available in the manufacturer software.

The reduction in orientation noise by FPM is even more clearly illustrated in maps of the grain reference orientation deviation (GROD) in Figure 4. The maps shown in the top row visualize the GROD angle and axis using a perceptually intuitive colour model as discussed in [39] and implemented in MTEX. In Figure 4a and b, the colours are scaled to full saturation at a misorientation angle of $0.25^{\circ}$; pole figure projections of the misorientation axes are

This article is protected by copyright. All rights reserved. 
plotted as black dots on the colour keys in Figure $4 \mathrm{c}$ and $\mathrm{d}$ in the sample coordinate system. We see that the RA result in Figure 4a again shows much more misorientation noise than the FPM result in Figure 4b. These results can be compared with the features in the KAM maps, shown in Figure $3 b$ and $f$ (for the RA result and FPM result respectively). Effects of topography are also just visible in the RA result, particularly in the region marked in Figure $4 a$. Figure $2 c$ shows that this triangular region contains two facets tilted away from the rest of grain. Topography artefacts from this region are not seen in the FPM misorientation map in Figure 4b.

In order to analyse the influence of various parameters in the EBSD system software, Figure 5 shows KAM maps of the [0001] grain indexed using HT and RA methods with varying Hough resolutions and number of detected bands. These settings are used to evaluate the resulting effect on the final orientation data, but they are not necessarily recommended for accurate practical measurements. Band centre detection was used throughout for this analysis. Apart from the subgrain near the bottom edge, the crystallographic features in this grain are below the noise floor of HT and RA indexing precision, and comparison with the FPM KAM map (Figure 3f) confirms this. Therefore, most features in the measured KAM maps in Figure 5 are taken to be noise or measurement artefacts.

Figure $5 \mathrm{a}$ and $\mathrm{b}$ were indexed using conventional $\mathrm{HT}$ analysis with band centre detection at 90 pixels Hough resolution, where orientations are solved from 8 and 12 detected bands respectively. Figure $5 \mathrm{a}$ is different to Figure $3 \mathrm{a}$, which uses band edge detection at 60 pixels Hough resolution. Orientation measurements using 8 bands are more discretised than those using 12 bands, as the solution has fewer degrees of freedom. This leads to patchy regions of $0^{\circ}$ measured KAM when using 8 bands, shown as white in Figure $5 \mathrm{a}$, compared to a more uniform orientation noise background from 12 bands. The number of bands used does not affect the misorientation patterning in the KAM maps.

Figure $5 c-f$ were indexed using the RA method, using either 8 or 12 bands at Hough resolutions of 90 or 250 pixels. The KAM values do not change much with Hough resolution, which shows that peak localisation is not a limiting factor in orientation precision for RA. The peak KAM value in the histograms are lower in RA compared to HT indexing (Figure 5a and b). Surface topography artefacts from ion beam polishing are visible in the KAM maps solved from 8 bands (Figure $5 c$ and e), but not from 12 bands (Figure $5 d$ and f). Due to the extra degrees of freedom in the RA approach, it can be speculated that e.g. the details of the pattern background processing in the presence of topography influence the RA result when a lower number of bands is considered and the corresponding effects are not averaged out over more bands. 


\section{Comparison with $\mathrm{ECCl}$}

In Figure 6, we compare an ECCl (b) with a map of the misorientation angle relative to the mean orientation in the red square in (a). This reveals the correspondence of many of the small features visible in the EBSD maps with dislocations visible in the ECCI. A few of these features are indicated by arrows to guide the eye. The line of 4 dislocations near the red square in Figure 6a allows us to estimate that angular changes of $0.03^{\circ}(0.5 \mathrm{mrad})$ can be resolved with about $100 \mathrm{~nm}$ spatial resolution in the current sample. These values have been estimated using MTEX by calculating the misorientation of nearby map points in the disclocation features. In comparison to the qualitative $\mathrm{ECCl}$, the quantitative EBSD data shows clearly a reduced spatial resolution, which can be seen by comparing the dislocation features marked by the arrows EBSD dislocation contrast features are sharp along the $x_{s}$ direction parallel to the tilt axis, but smeared out along $y_{s}$ in Figure $6 a$, most likely due to the anisotropic electron probe volume at the $70^{\circ}$ stage tilt used for EBSD [40].

The ion beam polishing procedure produced topography which is visible in the ECCI (Figure $6 b)$, most significantly as one ridge which arcs from the centre at the top of the image to midway down on the right and a second ridge from this arc, running down and left. This topography clearly caused variation in the correlation coefficient (Figure 2d) but has no visible effect on the resulting KAM and GROD angle maps (Figure $7 b$ and $d$ ). This suggests that the optimal orientations derived from the pattern matching approach are relatively stable against the effects of topography and image quality on the absolute value of the optimal correlation coefficient. Within the orientation resolution of our analysis, we do not observe orientation features that can be correlated with the large-scale topographical features in the NCC map shown in Figure $2 \mathrm{~d}$.

The dislocations visible in $\mathrm{ECCl}$ of the [0001] grain (Figure 6b) largely appear as points, indicating that they propagate at a high angle to the surface. This is consistent with previous TEM studies which have shown that the slip planes for WC are the prismatic $\{1 \overline{1} 00\}$ planes with the most commonly observed dislocations in WC grains, in as-sintered WC-Co, having Burgers vectors of $\frac{1}{3}\langle 11 \overline{2} 3\rangle, \frac{1}{3}\langle 11 \overline{20}\rangle$ and [0001] [33], [41], [42].Dislocations propagating at high angle to the surface are also observed in the $\mathrm{ECCl}$ in Figure 7a from a [11-20] grain. In addition, curved and linear features are also observed, indicating defects lying in the plane of the surface. On comparing the ECCl image with the EBSD maps indexed by FPM (Figure 7bd), it is clear that the misorientations produced by the defects are discernible in almost all of the EBSD maps, but particularly in the KAM and GROD axis map. This observation points to the role of specific dislocation types in producing the observed orientation changes. 


\section{Discussion}

\section{Angular resolution improvement of FPM}

As we have seen in the results above, the FPM approach to orientation refinement leads to significantly improved orientation precision as compared to the original orientation data obtained from the manufacturer system software. This quantitative improvement leads to a qualitative change in the kind of features which can be observed in the EBSD data, as is illustrated by the visibility of dislocation features in the EBSD orientation data. While the EBSD data lacks the spatial resolution possible with $\mathrm{ECCl}$, it provides valuable quantitative information for the interpretation of the ECCl features. From Figure 6, we can estimate the that the FPM approach reliably reveals orientational changes of the order of $0.03^{\circ}$ with about $100 \mathrm{~nm}$ spatial resolution for the WC samples which we investigated. In contrast, the base KAM values in the featureless regions of the RA results seen in Figure $3 \mathrm{~b}$ show minimum values between approximately $0.03^{\circ}$ and $0.1^{\circ}$. This means that the orientation noise level in the system data is significantly higher than the actual orientation changes in the features which are known from the FPM result. Thus, the fine orientation features are obliterated by noise in the conventional analysis of the measured EBSPs, but these features are clearly revealed by the FPM analysis on the stored EBSPs. The improvement of orientation precision, as described by values of the $95^{\text {th }}$ percentile of the KAM angle distribution, with $\vartheta_{95}=0.06^{\circ}$ is consistent with previous pattern matching applications on $\mathrm{Si}$ crystals [14]. The spatial resolution of about $100 \mathrm{~nm}$ is consistent with observations for Tungsten as discussed in [43]. We observe that the orientation precision of the FPM method presented here approaches the level of HR transmission Kikuchi (TKD) measurements on the strain field of single dislocations [44], where the spatial resolution of TKD is about an order of magnitude better than EBSD. The possibility to resolve fine orientational features by the FPM method demonstrated here suggests future investigations of additional strain effects via a projective pattern matching approach that has been used for mapping the local tetragonality in martensite using low-resolution patterns [45].

Our investigation indicates that the main information can even be obtained from pattern resolutions of $320 \times 240$ pixels, indicating that, in the present case, the angular resolution at full pattern resolution is limited not by the pixel size but by hardware limits, e.g. by optical aberrations in the EBSD detector, and by limits in the simulation model. The minimization of the optimal pattern size would be beneficial in terms of the data storage requirements for the saved raw data that is necessary for the pattern matching approach, as long as the electron dose per point is maintained, e.g. by EBSP frame averaging. We note that FPM of even standard pattern resolutions of $160 \times 120$ leads to a significant improvement of orientation precision, as has been shown in [13].

The practicality of the FPM approach depends on the speed at which the quantitative comparison of the experimental patterns with simulations at the same resolution can be carried out. We have optimized the corresponding calculations using GPU hardware 
acceleration, so that maps with resolutions in the order of $120 \times 150$ map points and $640 \times$ 480 pattern resolution as used here can be analysed typically within a few hours on a suitable PC. In this work, a GeForce RTX 2070 GPU and all 8 cores of an AMD Ryzen $72700 X$ @ $3700 \mathrm{MHz}$ CPU were used to produce the FPM results. In terms of speed, the FPM approach thus still cannot compete with the real-time performance provided by the Houghbased approaches in the manufacturer software. From a different point of view, one can also expect that the possible precision of Hough-transform based approaches could in principle be enhanced considerably by modified data analysis algorithms adapted to higher Hough resolutions, e.g. in cases where the reduced speed of high-precision data reanalysis is acceptable.

\section{Software orientation filtering artefacts}

As discussed in the introduction, various orientation filtering approaches have been used to improve the orientation precision of the raw orientation data derived from Hough-based data analysis procedures [3] [5] [7] [6]. It is thus pertinent to analyse the efficacy of the post-processing orientation filters in comparison to the FPM approach. If the postprocessing of noisy orientation data could deliver comparable results, this would circumvent the extra requirements of the FPM approach concerning data analysis time and computing power.

For example, as discussed above, the fine orientation features due to dislocations are masked by noise in the RA result as seen in Figure $3 a$ and Figure $3 b$. When filtering noisy orientation data, however, the underlying specific features are commonly unknown. A desirable filtering procedure would remove the noise and reveal the previously invisible and unknown orientation features. Because the FPM approach delivers high-precision orientation data with known orientation features which have been verified by $\mathrm{ECCl}$, we can use these optimized orientations as the ground truth against which to test the results of potential filtering approaches for the noisy data. Moreover, by filtering the optimized orientations themselves, we can determine filtering parameters which do not lead to an unacceptable loss of features from the ground truth result itself. In this way, the subsequent application of these filter parameters on the noisy data is known not to destroy the potential ground truth features which are initially hidden by the orientation noise.

In Figure 8, we show the result of this approach for the "infimal convolution" filter introduced in [7] and as implemented in MTEX 5.2. When we choose the filter parameters $\alpha=\beta=10^{-4}$, the dislocation features of the FPM result in Figure $8 \mathrm{a}$ are already significantly smoothed in (b), but still visible. This is why, for this example, we consider these filter settings as the maximum acceptable filter on the ground truth. In this way, we can assume that application of the same filter parameters on the noisy data will not blur the potential features more than seen in the result in Figure $8 \mathrm{~b}$. When we carry out the actual filtering of the original noisy RA data shown in Figure $8 \mathrm{c}$, we obtain the result seen in Figure $8 d$. Obviously, the filter parameters which have been determined as acceptable from the 
FPM result, have only a minor influence on the original noisy data, and, specifically, no new features are revealed from the remaining noise after orientation filtering. Moreover, we have also verified that stronger filtering leads to an increased smoothing of orientation features in the grain. To visualize the resulting loss of spatial resolution, in Figure 9 we show the filtering results assuming $\alpha=\beta=0.0075$ (this choice is based on [7], Fig.11) for both the RA data and the FPM refinement results. While the dislocation features are lost by this stronger filtering, it is reassuring that the infimal convolution filter consistently leads to very similar results for both types of orientation data, albeit at lower spatial resolution.

In contrast, the application of the Kuwahara filter on the two different starting data sets as shown in Figure 10 leads to considerably different results. In testing the action of the Kuwahara filter on the FPM reference data (Figure 10a and Figure 10d), we found that everything beyond a 1-neighbor Kuwahara filter will introduce severe artefacts into the data. This is shown by the results in Figure 10b and Figure 10e for the FPM reference data after filtering by a 3-neighbor Kuwahara filter as implemented in MTEX. On the other hand, applying the same filter to the noisy data from the manufacturer software still does not lead to a reduction of the KAM values to the size observed in experiment. Similar to what we have observed for the infimal convolution filter, this means that even stronger filtering would be necessary to reach the average KAM values of the FPM reference data. We can see in Figure 10c and Figure $10 \mathrm{f}$ that the 3-neighbor Kuwahara filtering of the original orientation data leads to the appearance of severe artefacts which have no real correspondence in the sample. Moreover, the artefacts are different for the two different starting data sets derived from the same EBSPs. In this respect, the infimal convolution filter creates more consistent results.

Summarizing our analysis concerning the filtering of orientation data, we find that a filtering option is not a substitute for an improved EBSP data analysis that derives more precise orientations from the same diffraction pattern data. Because the spatial orientation changes observed via EBSD are a key input into calculations of e.g. geometrically necessary dislocation densities, any orientation noise will increase the lower limit of the observable dislocation densities. It is important to observe that the need to filter noisy orientation data arises almost naturally for measurements which try to resolve features near the resolution limits of the EBSD method. With step sizes of a few tens of nanometers and feature sizes (spatial orientation changes) of the same order, a loss of spatial resolution by filtering cannot be excluded, because some degree of correlation between the true orientations of neighbouring map points is assumed in the filtering operation. While the infimal convolution filter makes the concomitant loss of spatial resolution transparent, the results for the Kuwahara filter showed that filtering noisy orientation data can introduce severe artefacts, which have no relation to any real features. However, smoothing of orientation data can be justified if the step size in the orientation map is sufficiently smaller than the typical feature sizes in the map. As dislocation features are observed at the lower limit of the possible EBSD 
resolution, however, this condition is hard to fulfil and filtering will often lead to a loss of spatial resolution, if not to the creation of artefacts.

\section{Misorientation artefacts in RA indexing}

We have compared misorientation patterning artefacts in RA and HT indexing methods. Figure 5 shows that the orientation precision of RA is better than HT within a uniform region such as a single facet in the grain. However, the extra degree of freedom in RA band detection makes it more susceptible to solution convergence artefacts when indexing using fewer Kikuchi bands, which leads to surface topography artefacts in Figure $5 c$ and e. Although $\mathrm{HT}$ indexing has a lower orientation precision, it is more robust to topography and convergence artefacts, and less likely to produce misleading KAM structures when mapping samples with high surface roughness, or poor pattern quality with few high contrast Kikuchi bands.

In contrast, FPM uses full-field EBSP intensities to find orientations, therefore it is much less affected by measurement uncertainty in band localisation. This can be seen in Figure $3 c-f$, where surface topography features are not visible in the KAM maps at any level of pixel binning.

\section{Summary}

We have presented a high-precision refinement of EBSD orientations using simulated pattern matching at the full available detector resolution. This allowed us to image the internal structure inside WC grains, revealing small orientation changes due to dislocations and subgrains, as verified by $\mathrm{ECCl}$. The correlation of qualitative $\mathrm{ECCl}$ features with quantitative orientation data from pattern matching will be advantageous for identification of specific dislocation types by ECCI. Concerning the effects of orientation filters on unrefined noisy data we demonstrate the ensuing loss of spatial resolution needs to be considered. Denoising of orientation data to obtain smooth gradients at best only reduces the spatial resolution, but there is a clear danger of creating artefacts. Concerning the question of de-noising or smoothing of EBSD data, it can therefore be argued, that before applying such measures, all the possible information should be extracted which is available without such filtering techniques. Filtering should not be substitute for an improved orientation analysis based on the raw Kikuchi pattern data.

\section{Acknowledgements}

Aimo Winkelmann was supported by the Polish National Agency for Academic Exchange (NAWA), grant no. PPN/ULM/2019/1/00068/U/00001. Carol Trager-Cowan would like to acknowledge financial support of the Engineering and Physical Sciences Research Council, 
UK via Grant No. EP/P015719/1. "Quantitative non-destructive nanoscale characterisation of advanced materials".

Data associated with this research are available at [DOI to be inserted here].

References

[1] A. J. Schwartz, M. Kumar, B. L. Adams and D. P. Field, Eds., Electron Backscatter Diffraction in Materials Science, Springer US, 2009.

[2] F. J. Humphreys, "Quantitative metallography by electron backscattered diffraction," J. Microsc., vol. 195, pp. 170-185, 1999.

[3] F. J. Humphreys, "Grain and subgrain characterisation by electron backscatter diffraction," J. Mater. Sci., vol. 6, pp. 3833-3854, 2001.

[4] F. J. Humphreys, P. S. Bate and P. J. Hurley, "Orientation averaging of electron backscattered diffraction data," Journal of Microscopy, vol. 201, pp. 50-58, 2001.

[5] D. Chen and J.-C. Kuo, "Bilateral filter based orientation smoothing of EBSD data," Ultramicroscopy, vol. 110, pp. 1297-1305, 2010.

[6] A. Seret, C. Moussa, M. Bernacki, J. Signorelli and N. Bozzolo, "Estimation of geometrically necessary dislocation density from filtered EBSD data by a local linear adaptation of smoothing splines," Journal of Applied Crystallography, vol. 52, pp. 548-563, 52019.

[7] R. Hielscher, C. B. Silbermann, E. Schmidl and J. Ihlemann, "Denoising of crystal orientation maps," Journal of Applied Crystallography, vol. 52, pp. 984-996, 2019.

[8] S. Wright, M. Nowell, S. Lindemann, P. Camus, M. De Graef and M. Jackson, "Introduction and comparison of new EBSD post-processing methodologies," Ultramicroscopy, vol. 159, pp. 81-94, 2015.

[9] A. Wilkinson, D. Collins, Y. Zayachuk, R. Korla and A. Vilalta-Clemente, "Applications of multivariate statistical methods," Ultramicroscopy, vol. 196, pp. 88-98, 2019.

[10] F. Ram and M. De Graef, "Phase differentiation by EBSD using the dictionary indexing approach,” Acta Mater., vol. 144, pp. 352-364, 2018.

[11] Y. H. Chen, S. U. Park, G. Wei, G. Newstadt, M. A. Jackson, J. P. Simmons, M. De Graef and A. O. Hero, "A dictionary approach to EBSD indexing," Microsc.Microanal., vol. 21, pp. 739-752, 2015.

[12] G. Nolze, R. Hielscher and A. Winkelmann, "Electron backscatter diffraction beyond the mainstream," Crystal Research and Technology, vol. 52, p. 1600252, 102016.

This article is protected by copyright. All rights reserved. 
[13] G. Nolze, M. Jürgens, J. Olbricht and A. Winkelmann, "Improving the precision of orientation measurements from technical materials via EBSD pattern matching," Acta Materialia, vol. 159, pp. 408-415, 2018.

[14] T. Friedrich, A. Bochmann, J. Dinger and S. Teichert, "Application of the pattern matching approach for EBSD calibration and orientation mapping," Ultramicroscopy, vol. 184, pp. 44-51, 2018.

[15] A. Nicolaÿ, J. M. Franchet, J. Cormier, H. Mansour, M. D. Graef, A. Seret and N. Bozzolo, "Discrimination of dynamically and post-dynamically recrystallized grains based on EBSD data: application to Inconel 718," Journal of Microscopy, vol. 273, pp. 135-147, 112018.

[16] A. Winkelmann, G. Nolze, S. Vespucci, G. Naresh-Kumar, C. Trager-Cowan, A. Vilata-Clemente, A. Wilkinson and M. Vos, "Diffraction effects and inelastic electron transport in angle-resolved microscopic imaging applications," J.Microsc., vol. 267, pp. 330-346, 2017.

[17] A. J. Wilkinson, G. Meaden and D. J. Dingley, "High-resolution elastic strain measurement from electron backscatter diffraction patterns: new levels of sensitivity.," Ultramicroscopy, vol. 106, pp. 307-13, 32006.

[18] A. J. Wilkinson, D. J. Dingley and G. Meaden, "Strain Mapping Using Electron Backscatter Diffraction," in Electron Backscatter Diffr. Mater. Sci., 2 ed., A. J. Schwartz, M. Kumar, B. L. Adams and D. P. Field, Eds., Boston, MA: Springer US, 2009.

[19] T. B. Britton, I. Holton, G. Meaden and D. Dingley, "High angular resolution electron backscatter diffraction: measurement of strain in functional and structural materials," Microscopy and Analysis, vol. 27, 2013.

[20] T. B. Britton, C. Maurice, R. Fortunier, J. H. Driver, A. P. Day, G. Meaden, D. J. Dingley, K. Mingard and A. J. Wilkinson, "Factors affecting the accuracy of high resolution electron backscatter diffraction when using simulated patterns.," Ultramicroscopy, vol. 110, pp. 1443-53, 112010.

[21] A. J. Wilkinson and P. B. Hirsch, "Electron diffraction based techniques in scanning electron microscopy of bulk materials," Micron, vol. 28(4), pp. 279-308, 1997.

[22] M. A. Crimp, B. A. Simkin and B. C. Ng, "Demonstration of the $g \cdot b x u=0$ edge dislocation invisibility criterion for electron channelling contrast imaging," Philos.Mag.Lett., vol. 81(12), pp. 833-837, 2001.

[23] C. Trager-Cowan, F. Sweeney, P. W. Trimby, A. P. Day, A. Gholinia, N.-H. Schmidt, P. J. Parbrook, A. J. Wilkinson and I. M. Watson, "Electron backscatter diffraction and electron channeling contrast imaging of tilt and dislocations in nitride thin films," Phys.Rev.B, vol. 75(8), p. 085301, 2007.

This article is protected by copyright. All rights reserved. 
[24] Y. Picard, R. Kamaladasa, M. De Graef, N. Nuhfer, W. Mershon, T. Owens, L. Sedlacek and F. Lopour, "Future Prospects for Defect and Strain Analysis in the SEM via Electron Channeling," Micros.Today, vol. 20(2), pp. 12-16, 2012.

[25] S. Zaefferer and N. N. Elhami, "Theory and application of electron channelling contrast imaging under controlled diffraction conditions," Acta Mater, vol. 75, pp. 20-50, 2014.

[26] H. Mansour, J. Guyon, M. A. Crimp, N. Gey, B. Beausir and N. Maloufi, "Accurate electron channeling contrast analysis of dislocations in fine grained bulk materials," Scripta Materialia, Vols. 84-85, pp. 11-14, 82014.

[27] J. Guyon, H. Mansour, N. Gey, M. A. Crimp, S. Chalal and N. Maloufi, "Sub-micron resolution selected area electron channeling patterns.," Ultramicroscopy, vol. 149, pp. 34-44, 2015.

[28] S. Carnevale, J. Deitz, J. Carlin, Y. Picard, M. D. Graef, S. A. Ringel and T. J. Grassman, "Rapid misfit dislocation characterization in heteroepitaxial III-V/Si thin films by electron channeling contrast imaging," Appl. Phys. Lett, vol. 104(23), p. 232111, 2014.

[29] R. J. Kamaladasa, F. Liu, L. Porter, R. Davis, D. Koleske, G. Mulholland, K. Jones and Y.N. Picard, "Identifying threading dislocations in GaN films and substrates by electron channelling," J. Microsc., vol. 244(3), pp. 311-319, 2011.

[30] M. Ben Saada, N. Gey, B. Beausir, X. Iltis, H. Mansour and N. Maloufi, "Sub-boundaries induced by dislocational creep in uranium dioxide analyzed by advanced diffraction and channeling electron microscopy," Materials Characterization, vol. 133, pp. 112-121, 112017.

[31] H. Mansour, M. A. Crimp, N. Gey, X. Iltis and N. Maloufi, "Dislocation analysis of a complex subgrain boundary in UO_2 ceramic using accurate electron channelling contrast imaging in a scanning electron microscope," Ceramics International, vol. 45, pp. 18666-18671, 102019.

[32] J. García, V. C. Ciprés, A. Blomqvist and B. Kaplan, "Cemented carbide microstructures: a review," International Journal of Refractory Metals and Hard Materials, vol. 80, pp. 40-68, 4 2019.

[33] B. M. Jablon, K. P. Mingard, A. Winkelmann, G. Naresh-Kumar, B. Hourahine and C. TragerCowan, "Subgrain structure and dislocations in WC-Co hardmetals revealed by electron channelling contrast imaging," Int.J. Refractory Met. and Hard Mater., vol. 87, p. 105159, 2020.

[34] K. Thomsen, N. H. Schmidt, A. Bewick, K. Larsen and J. Goulden, "Improving the Accuracy of Orientation Measurements using EBSD," Proc.Microsc.Microanal., vol. 19, no. S2, pp. 724-725, 2013.

[35] C. Maurice and R. Fortunier, "A 3D Hough transform for indexing EBSD and Kossel patterns," Journal of Microscopy, vol. 230, no. 3, pp. 520-529, 2008.

This article is protected by copyright. All rights reserved. 
[36] E. J. Kirkland, Advanced Computing in Electron Microscopy, Springer Science + Business Media, 2010.

[37] A. Winkelmann, T. B. Britton and G. Nolze, "Constraints on the effective electron energy spectrum in backscatter Kikuchi diffraction," Physical Review B, vol. 99, no. 6, p. 064115, 22019.

[38] X. Tao and A. Eades, "Another Way to Implement Diffraction Contrast in SEM," Microscopy Today, vol. 11, pp. 36-38, 42003.

[39] K. Thomsen, K. Mehnert, P. Trimby and A. Gholinia, "Quarternion-based disorientation colouring of orientation maps," Ultramicroscopy, vol. 182, pp. 62-37, 2017.

[40] D. Chen, J.-C. Kuo and W.-T. Wu, "Effect of microscopic parameters on EBSD spatial resolution," Ultramicroscopy, vol. 111, no. 9-10, pp. 1488-1494, 2011.

[41] B. Roebuck and E. A. Almond, "Deformation and Fracture Processes and the Physical Metallurgy of WC-Co Hardmetals," Int.Mater.Rev, vol. 33, pp. 90-112, 1988.

[42] M. K. Hibbs and R. Sinclair, "Room-temperature deformation mechanisms and the defect structure of tungsten carbide," Acta.Metall., vol. 29, pp. 1645-1654, 1981.

[43] A. Tripathi and S. Zaefferer, "On the resolution of EBSD across atomic density and accelerating voltage with a particular focus on the light metal magnesium," Ultramicroscopy, vol. 207, p. 112828, 122019.

[44] H. Yu, J. Liu, P. Karamched, A. J. Wilkinson and F. Hofmann, "Mapping the full lattice strain tensor of a single dislocation by high angular resolution transmission Kikuchi diffraction (HRTKD)," Scripta Materialia, vol. 164, pp. 36-41, 2019.

[45] A. Winkelmann, G. Nolze, G. Cios and T. Tokarski, “Mapping of local lattice parameter ratios by projective Kikuchi pattern matching," Physical Review Materials, vol. 2, p. 123803, 2018.

This article is protected by copyright. All rights reserved. 


\section{Figure Captions}

Figure 1. Example EBSP from the [0001] grain at full $640 \times 480$ resolution (top) compared to

A a simulated pattern (bottom). Patterns have been background corrected and normalized to zero mean and unit standard deviation. NCC coefficient $r=0.69$.



This article is protected by copyright. All rights reserved. 
Figure 2

a) SEM image based on intensity of the saved Kikuchi patterns, showing the topography of the WC grains and the Co binder phase.

b) Inverse Pole Figure Map (IPF-Z), showing the direction of the surface normal Z in the crystal coordinate system. The red color indicates that the central grain is oriented approximately with [0001] along the surface normal.

c) ECCI micrograph, showing the presence of dislocation features

d) Map of the NCC coefficient between the measured pattern at each map point and the best-fit simulated pattern.
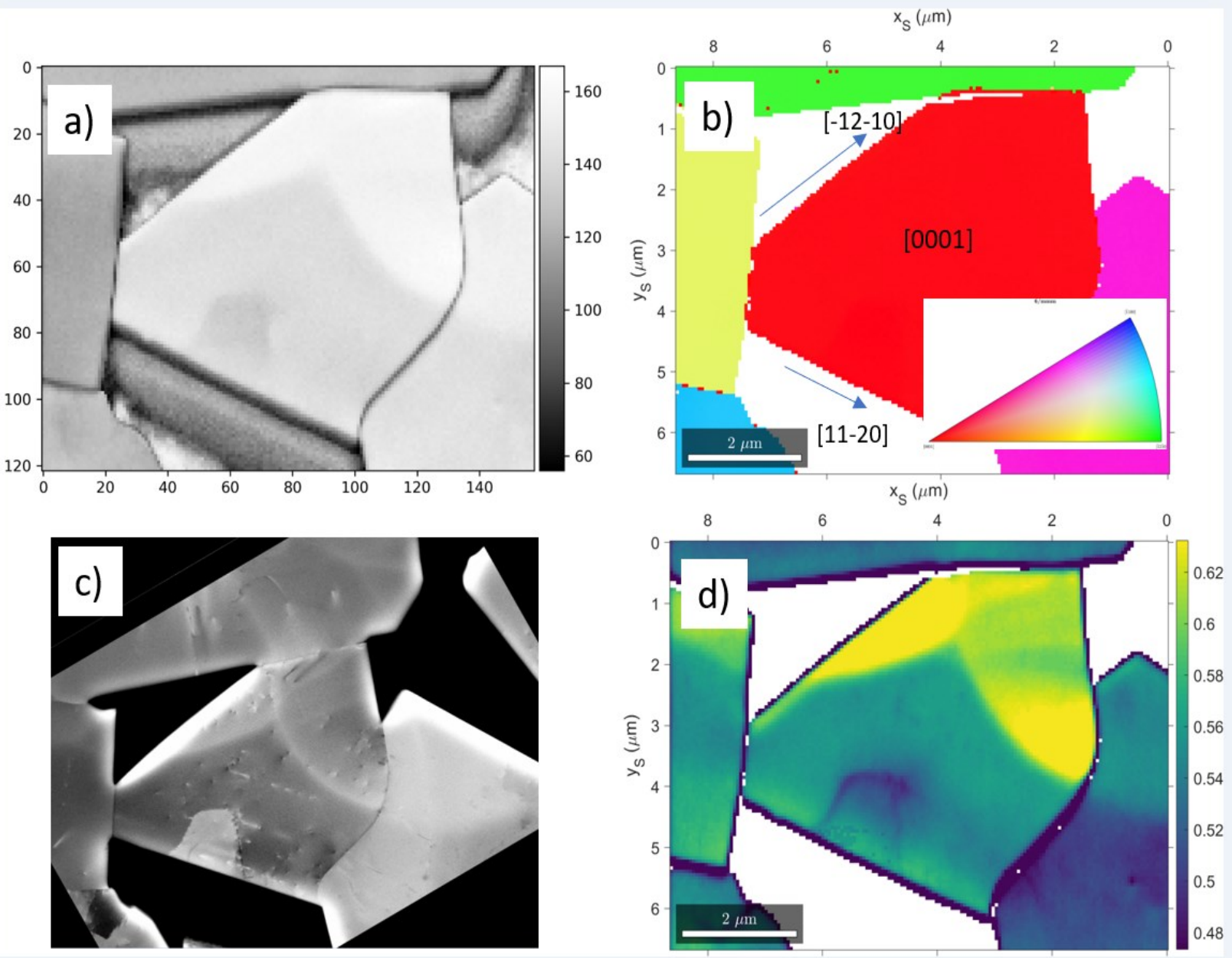

This article is protected by copyright. All rights reserved. 
Figure 3: Estimation of the local orientation variations resulting from different EBSD data analysis approaches. The Kernel Average Misorientation (KAM) angle is shown for a) the standard Hough-based orientations of the manufacturer software, b) Refined Accuracy results from the manufacturer software c)-f) FPM orientation refinement with pattern resolutions from $80 \times 60$ to $640 \times 480$. g) Histograms of the KAM angles for the grain shown in a)-f), together with the $95^{\text {th }}$ percentile for an estimation of the relative precision between the different analyses.
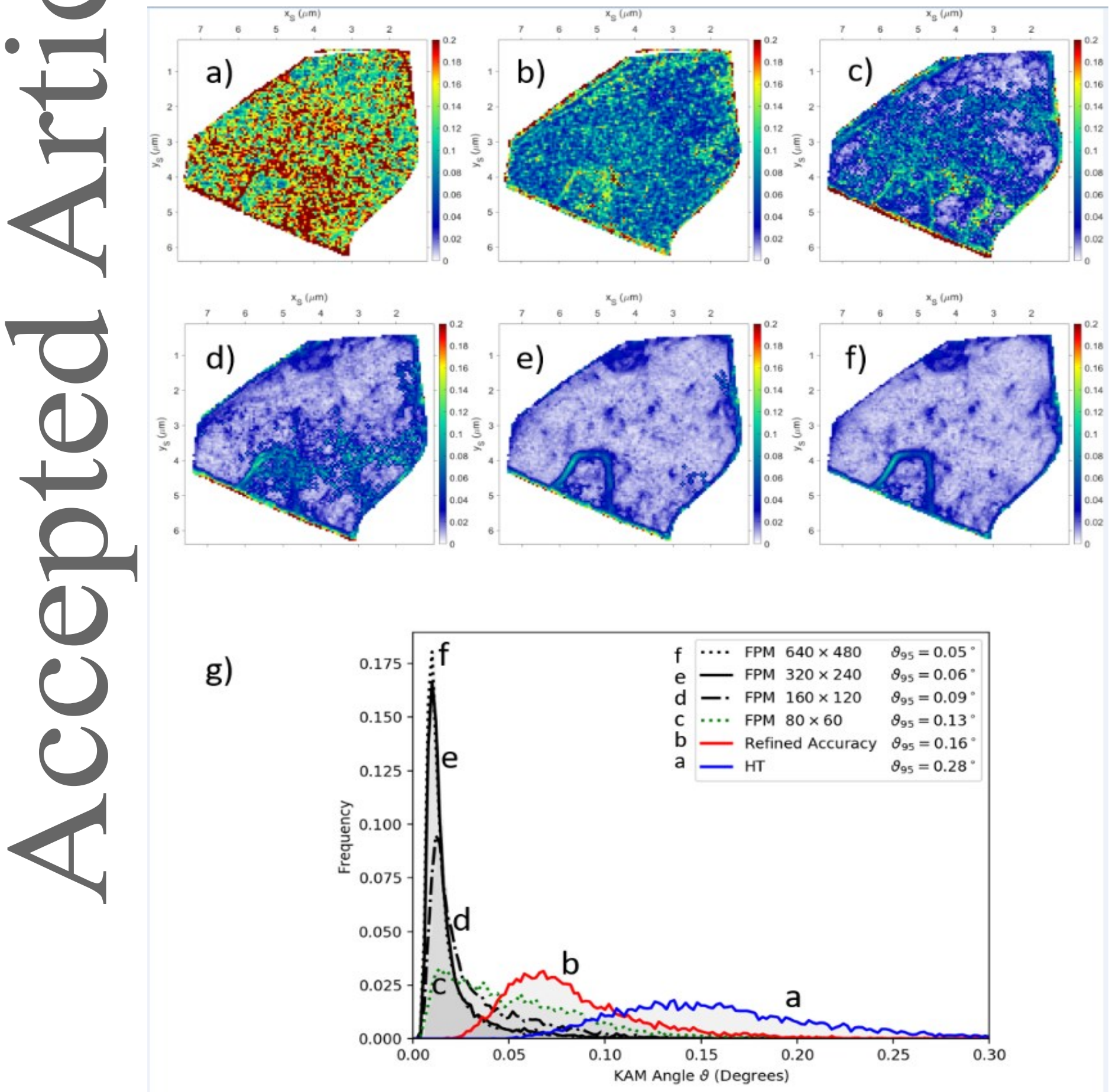

This article is protected by copyright. All rights reserved. 
Figure 4: Comparison of the Refined Accuracy data (left) and pattern matching results for $640 \times 480$ (right). (a-d): Maps and color keys of the grain reference orientation deviation (GROD, axis and angle) with the mean orientation of the grain as the reference. The dashed lines in a) indicate topographic features produced by ion beam polishing which influence the results of the RA method, compare also Figure 1. The color keys correspond to the disorientation colouring introduced in [39].
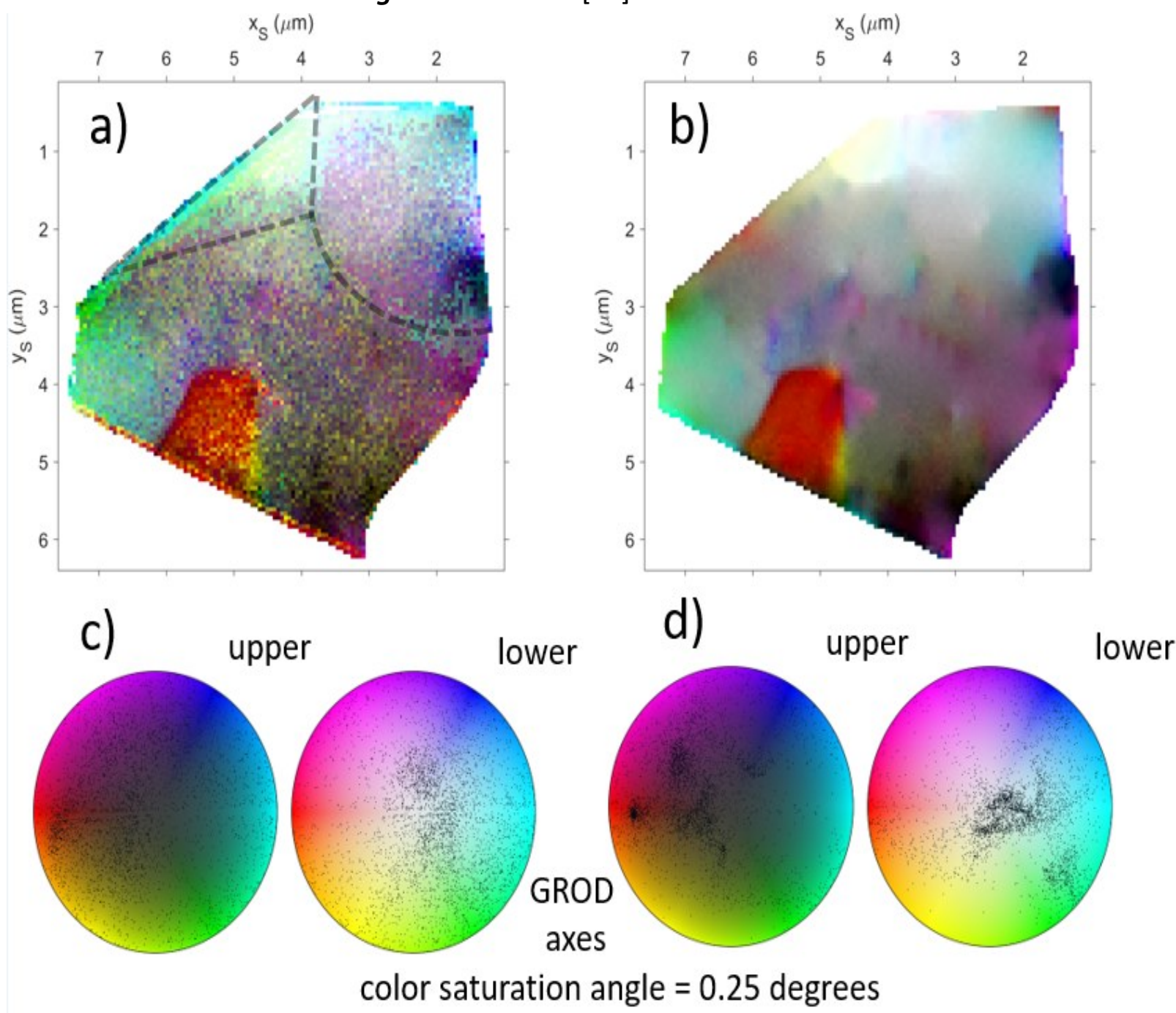
Figure 5: KAM maps showing the effect of Hough resolution and number of detected bands on orientation artefacts. a), b) Conventional Hough transform indexing at 90 pixels Hough resolution, using 8 and 12 bands respectively. c), d) Refined accuracy indexing at 90 pixels Hough resolution, using 8 and 12 bands respectively; e), f) Refined accuracy indexing at 250 pixels Hough resolution, using 8 and 12 bands respectively. Band centre detection was used throughout. Inset plots show KAM histogram distributions between 0 and $0.3^{\circ}$, with consistent axis scaling between subplots.

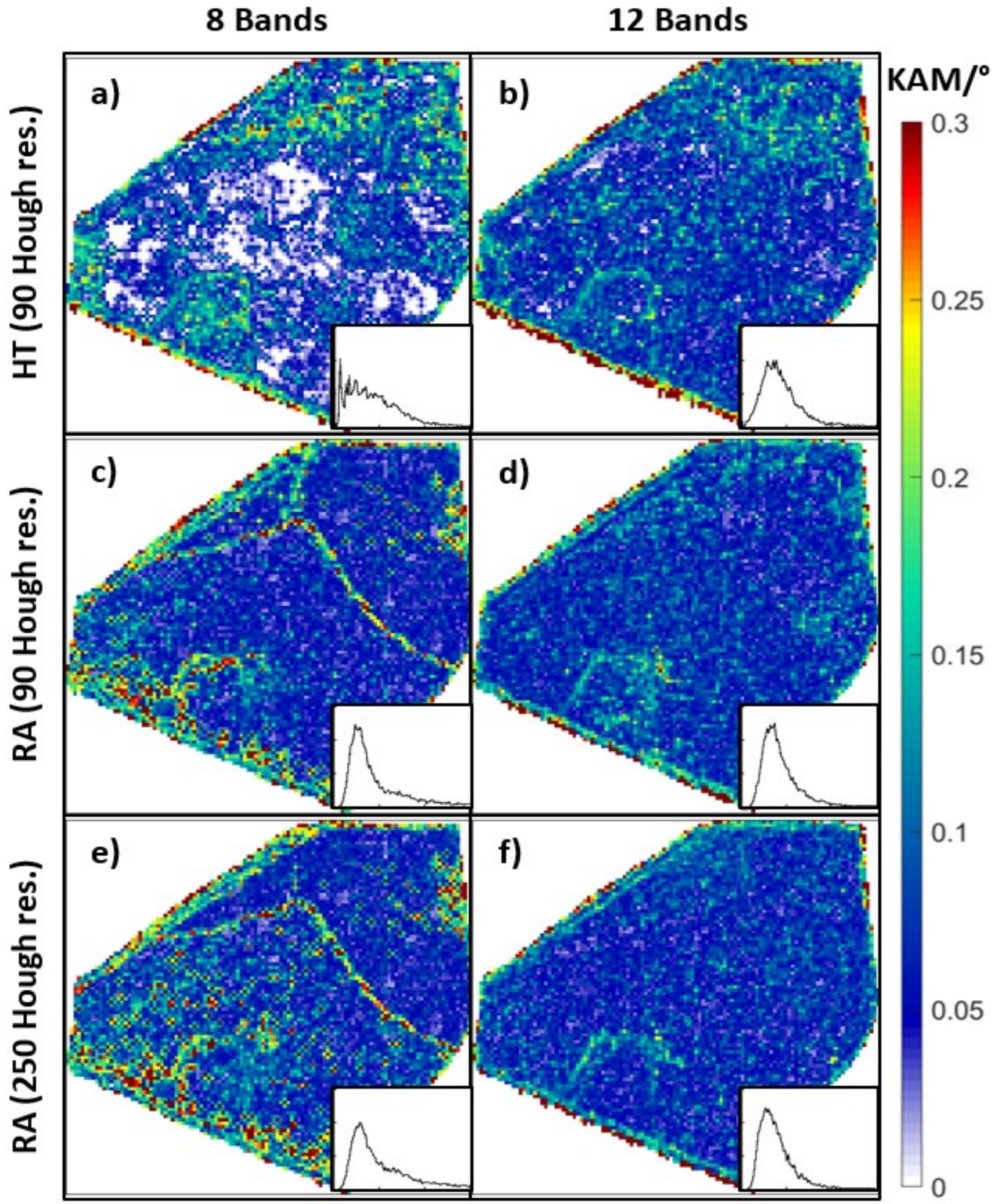

This article is protected by copyright. All rights reserved. 
Figure 6. Quantification of ECCI features by FPM EBSD results. Features in the ECCI micrograph (b) are compared to the map of the misorientation angle (in degrees) relative to the mean orientation in the marked square in (a). The feature of 4 dislocations near the red square allows to estimate that angular changes of $0.03^{\circ}(0.5 \mathrm{mrad})$ can be resolved with about $100 \mathrm{~nm}$ spatial resolution.



This article is protected by copyright. All rights reserved. 
Figure 7: Comparison of ECCI (a), KAM angle (b), and GROD axis (c) and angle (d) relative to the mean orientation in the region marked by the red square. This grain has its surface normal perpendicular to [0001] and [10-10] as shown in c). The elongated grain shape in the EBSD data compared to $\mathrm{ECCl}$ is an image drift artefact due to the long acquisition time.
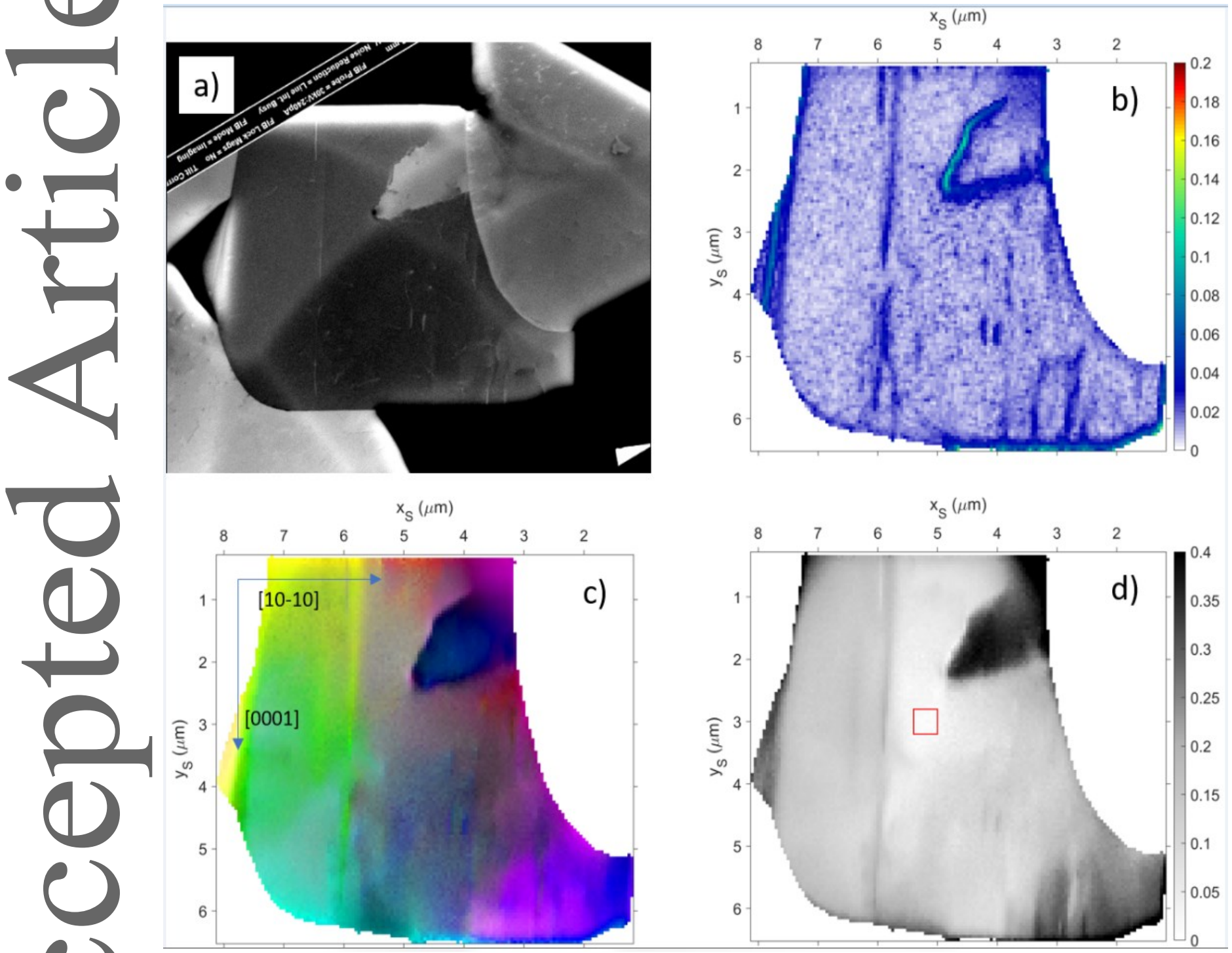
Figure 9: Effect of stronger filtering using the infimal convolution filter. For a choice of $\alpha, \beta=0.0075$, both the results of the FPM (left column) and the RA analysis (right column) are very similar and show a reduced spatial resolution.




Figure 10: Appearance of artefacts when filtering noisy orientation data using a Kuwahara filter. Upper row (a-c): GROD axis-angle maps, lower row (d-f): KAM angle maps. The reference, unfiltered, result is the best-fit FPM result shown in a) and d). We apply a 3neighbor Kuwahara filter as implemented in MTEX to the orientation data: $b, e)$ effect on the FPM reference result, $c, f)$ : effect on the Refined Accuracy (RA) data. The comparison to the FPM results $(a, d)$ shows that severe artefacts are created both in the RA data $(c, f)$ and filtered FPM data $(b, e)$. Note that the application of the Kuwahara filter on different starting data leads to different filter results, i.e. different artefacts in both cases.
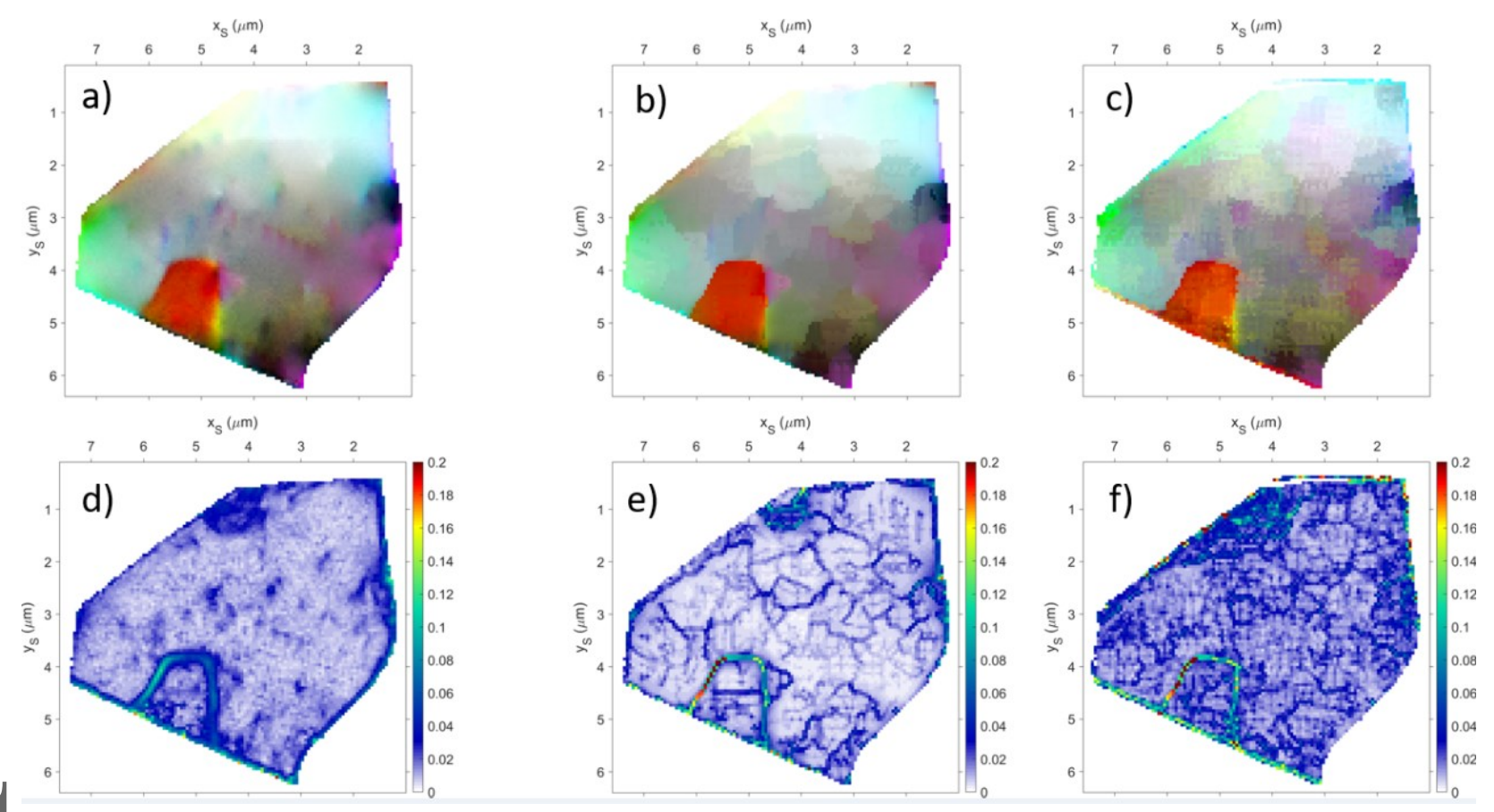